\section{ON RADIANT MATTER ${ }^{1}$}

TO throw light on the title of this lecture I must go back more than sixty years-to I8I6. Faraday, then a mere studen and ardent experimentalist, was twenty-four years old, and at this early period of his career he delivered a series of lectures on the general properties of matter, and one of them bore the remarkable title, "On Radiant Matter." The great philosopher's notes of this lecture are to be found in Dr. Bence Jones's "Life and Letters of Faraday," and I will here quote a passage in which he first employs the expression Radiant Matter:-

"If we conceive a change as far beyond vaporisation as that is above fluidity, and then take into account also the proportional increased extent of alteration as the changes rise, we shall perhaps, if we can form any conception at all, not fall far short of radiant matter ; and as in the last conversion many qualities were lost, so here also many more would disappear."

Faraday was evidently engrossed with this far-reaching speculation, for three years later-in 1819-we find him bringing fresh evidence and argument to strengthen his startling hypothesis. His notes are now more extended, and they show that in the intervening three years he had thonght much and deeply on this higher form of matter. He first points out that matter may be classed into four states-solid, liquid, gaseous, and radiant-these modifications depending upon differences in their several essential properties. He admits that the existence of radiant matter is as yet unproved, and then proceeds, in a series of ingenious analogical arguments, to show the probability of its existence, ${ }^{2}$

If, in the beginning of this century, we had asked, What is a gas? the answer then would have been that it is matter, expanded and rarefied to such an extent as to be impalpable, save when set in violent motion; invisible, incapable of assuming or of being reduced into any definite form like solids, or of forming drops like liquids; always ready to expand where no resistance is offered, and to contract on being subjected to pressure. Sixty years ago such were the chief attributes assigned to gases. Modern research, however, has greatly enlarged and modified our views on the constitution of these elastic fluids. Gases are now considered to be composed of an almost infinite number of small particles or molecules, which are constantly moving in every direction with velocities of all conceivable mag. nitudes. As these molecules are exceedingly numerous, it fol. lows that no molecule can move far in any direction without coming in contact with some other molecule. But if we exhaust the air or gas contained in a closed vessel, the number of molecules becomes diminished, and the distance through which any one of them can move without coming in contact with another is increased, the length of the mean free path being inversely pro. portional to the number of molecules present. The further this process is carried the longer becomes the average distance a molecule can travel before entering into collision; or, in other words, the longer its mean free path the more the physical properties of the gas or air are modified. Thus, at a certain point, the phenomena of the radiometer become possible, and on pushing the rarefaction still further, i.e., decreasing the number of

${ }^{x}$ A lecture delivered to the British Association for the Advancement of Science, at Sheffield, Friday, August 22, 1879, by William Crookes, F.R.S. " "I may now notice a curious progression in physical properties accompanying changes of form, and which is perhaps sufficient to induce, in the inventive and sanguine philosopher, a considerable degree of belief in the association of the radiant form with the others in the set of changes I have ciation of

"As we ascend from the solid to the fluid and gaseous states, physical properties diminish in number and variety, each state losing some of those properties diminish in number and variety, each state losing some of those which belonged to the preceding state. When solids are converted into
fluids, all the varieties of hardness and softness are necessarily lost. Crysfluids, all the varieties of hardness and softness are necessarily lost. Crys-
talline and other shapes are destroyed. Opacity and colour frequently give way to a colourless transparency, and a general mobility of particles is conferred.

"Passing

Passing onward to the gaseous state, still more of the evident characters of bodies are annihilated. The immense differences in their weight almost disappear; the remains of difference in colour that were left are lost. Transparency tecomes universal, and they are all elastic. They now form but ene substan, of elasticity, and form, which render the number of solids and fluids almost infinite, are now supplied by a $f$ nimportant shades of colour.

"To those, therefore, who admit the radiant form of matter, no difficulty exists in the simplicity of the properties it possesses, but rather an argumen in their favour. These persons show you a gradual resignation of propertie in the matter we can appreciate as the matter ascends in the scale of forms, and they would be suprised if that effect were to cease at the gaseous state. They point cut the greater exertions which nature makes at each step:of the change, and think that, consistently, it ought to be greatest in the passage from the gaseous to the radiant form." - Life and Letters of Fraraday, vol. i. p. 308 . molecules in a given space and lengthening their mean free path, the experimental results are obtainable to which I am now about to call your attention. So distinct are these phenomena from anything which occurs in air or gas at the ordinary tension, that we are led to assume that we are here brought face to face with matter in a fourth state or condition, a condition as far removed from the state of gas as a gas is from a liquid.

\section{Mean Fres Path. Radiant Matter}

I have long believed that a well-known appearance observed in vacuum tubes is closely related to the phenomena of the mean free path of the molecules. When the negative pole is examined while the discharge from an induction-coil is passing through an exhausted tube, a dark space is seen to surround it. This dark space is found to increase and diminish as the vacuum is varied, in the same way that the mean free path of the molecules lengthens and contracts. As the one is perceived by the mind's eye to get greater, so the other is seen by the bodily eye to increase in size; and if the vacuum is insufficient to permit mucb play of the molecules before they enter into collision, the pas. sage of electricity shows that the "dark space" has shrunk to small dimensions. We naturally infer that the dark space is the mean free path of the molecules of the residual gas, an inference confirmed by experiment.

I will encleavour to render this "dark space" visible to all present. Here is a tube (Fig. I) having a pole in the centre in

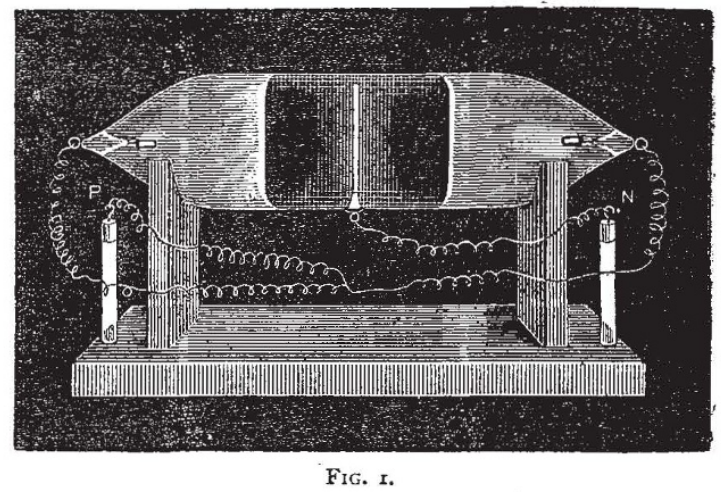

the form of a metal disk, and other poles at each end. The centre pole is made negative, and the two end poles connected together are made the positive terminal. The dark space will be in the centre. When the exhaustion is not very great the dark space extends only a little on each side of the negative pole in the centre. When the exhaustion is good, as in the tube before you, and I turn on the coil, the dark space is seen to extend for about an inch on each side of the pole.

Here, then, we see the induction spark actually illuminating the lines of molecular pressure caused by the excitement of the negative pole. The thickness of this dark space is the measure of the mean free path between successive collisions of the molecules of the residual gas. The extra velocity with which the negatively electrified molecules rebound from the excited pole, keeps back the more slowly moving molecules which are advancing towards that pole. A conflict occurs at the boundary of the dark space, where the luminous margin bears witness to the energy of the discharge.

Therefore the residual gas-or, as I prefer to call it, the: gaseous residue-within the dark space, is in an entirely different state to that of the residual gas in vessels at a lower degree of exbaustion. To quote the words of our last year's President, in his address at Dublin :-

"In the exhausted column we have a vehicle for electricity not constant like an ordinary conductor, but itself modified by the passage of the discharge, and perhaps subject to laws differing materially from those which it obeys at atmospheric pressure."

In the vessels with the lower deoree of exhaustion, the length of the mean free path of the molecules is exceedingly small as compared with the dimensions of the bulb, and the properties belonging to the ordinary gaseous state of matter, depending upon constant collisions, can be observed. But in the phenomenz now about to be examined, so high is the exhaustion carried that the dark snace around the negative pole has widened out till it entirely fills the tube. By great rarefaction the mean free path 
has become so long that the hits in a given time in comparison to the misses may be disregarded, and the average molecule is now allowed to obey its own motions or laws without interference. The mean free path, in fact, is comparable to the dimensions of the vessel, and we have no longer to deal with a continuous portion of matter, as would be the case were the tubes less highly exhausted, but we must here contemplate the molecules individually. In these highly exhausted vessels the molecules of the gaseous residue are able to dart across the tube with comparatively few collisions, and radiating from the pole with enormous velocity, they assume properties so novel and so characteristic as to entirely justify the application of the term borrowed from Faraday, that of Radiant Matter.

Radiant Matter exerts powerful Phosphorogenic Action where it strikes

I have mentioned that the radiant matter within the dark space excites luminosity where its velocity is arrested by residual gas outside the dark space. Bat if no residual gas is left, the molecules will have their velocity arrested by the sides of the glass; and here we come to the first and one of the most noteworthy properties of radiant matter discharged from the negative pole-its power of exciting phosphorescence when it strikes against solid matter. The number of bodies which respond luminously to this molecular bombardment is very great, and the resulting colours are of every variety. Glass, for instance, is highly phosphorescent when exposed to a stream of radiant matter. Here (Fig. 2) are three bulbs composed of different glass : one is uranium glass (a), which phosphoresces of a dark green colour; another is English glass (b), which phosphoresces of a blue colour; and the third $(c)$ is soft German glass-of which most of the apparatus before you is made-which phos. phoresces of a bright apple-green.

My earlier experiments were almost entirely carried on by the aid of the phosphorescence which glass takes up when it is under the infuence of the radiant discharge; but many other substances possess this phosphorescent power in a still higher degree than glass. For instance, here is some of the luminous sulphide of calcium prepared according to M. Ed. Becquerel's description. When the sulphide is exposed to light--even candle-light-it phosphoresces for hours with a bluish-white colour. It is, however, much more strongly phosphorescent to the molecular discharge in a good vacuum, as you will see when I pass the discharge through this tube.

Other substances besides English, German, and uraniwu glass, and Becquerel's luminous sulphides, are also phosphorescent.

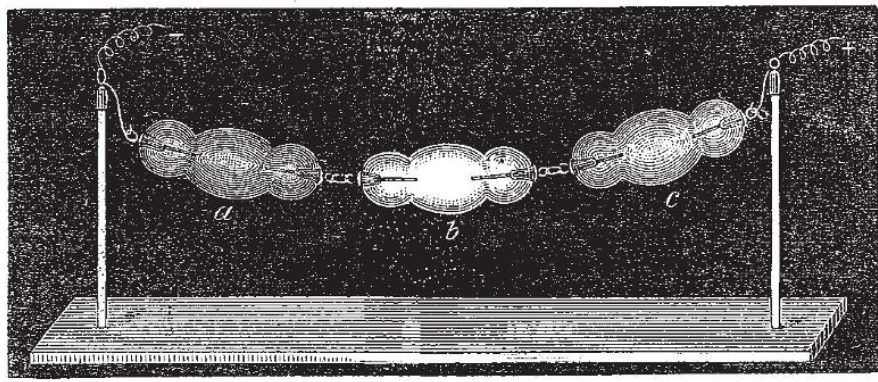

FIG. 2 ,

The rare mineral phenakite (aluminate of glucinum) phosphoresces blue; the inineral spodumene (a silicate of aluminium and lithium) phosphoresces a rich golden yellow; the emerald gives out a crimson light. But without exception, the diamond is the most sensitive substance I have yet met for ready and brilliant phosphorescence. Here is a very curious fluorescent diamond,

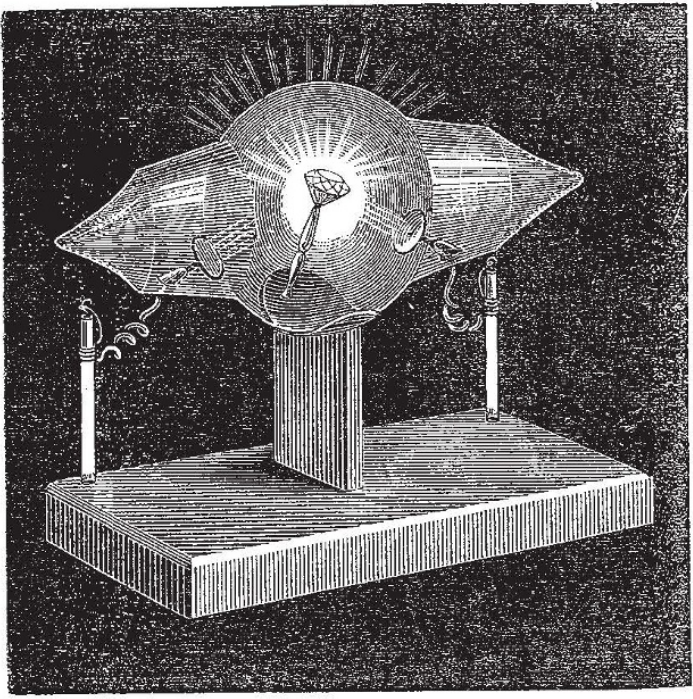

Fig, 3.

green by daylight, colourless by candle-light. It is mounted in the centre of an exhausted bulb (Fig. 3), and the molecular discharge will be directed on it from below upwards. On darkening the room you see the diamond shines with as much light as a candle, phosphorescing of a bright green.
Next to the diamond the ruby is one of the most remarkable stones for phosphorescing. In this tube (Fig. 4) is a fine collection of ruby pebbles. As soon as the induction-spark is turned on you will see these rubies shining with a brilliant rich red tone, as if they were glowing hot. It scarcely matters what colour the ruby is, to begin with. In this tube of natural rubies there are stones of all colours-the deep red and also the pale pink ruby. There are some so pale as to be almost colourless, and some of the highly-prized tint of pigenn's blood; but under the impact of radiant matter they all phosphoresce with about the sime colour.

Now the ruby is nothing but crystallised alumina with a little

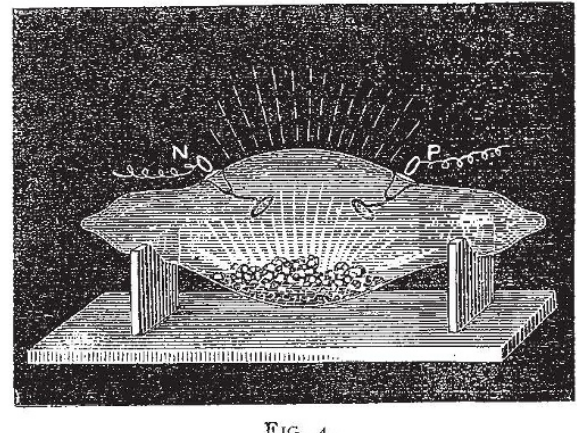

FIG. 4 .

colouring-matter. In a paper by Ed. Becquerel, ${ }^{1}$ published twenty years ago, he describes the appearance of alumina as vlowing with a rich red colour in the phosplooroscope. Here is some precipitated alumina prepared in the most careful manner. It has been heated to whiteness, and you see it also glows under the molecular discharge with the same rich red colour.

The spectrum of the red light emitted by these varieties of alumina is the same as described by Becquerel twenty years ago. There is one intense red line, a little helow the fixed line $B$ in

${ }^{1}$ Antales de Chintie et de Physique, 3rd series, vol. Ivii., p. 50, 1859.". il 
the spectrum, having a wave-length of about 6895 . There is a continuous spectrum beginning at about $\mathrm{B}$, and a few fainter lines beyond it, but they are so faint in comparison with this red line that they may be neglected. This line is easily seen by examining with a small pocket spectroscope the light reflected from a good ruby.

There is one particular degree of exhaustion more favourable than any other for the development of the properties of radiant matter which are now under examination. Roughly speaking, it may be put at the millionth of an atmosphere. ${ }^{1}$ At this degree of exhaustion the phosphorescence is very strong, and after that it begins to diminish until the spark refuses to pass. ${ }^{2}$

I have here a tube (Fig. 5) which will serve to illustrate the

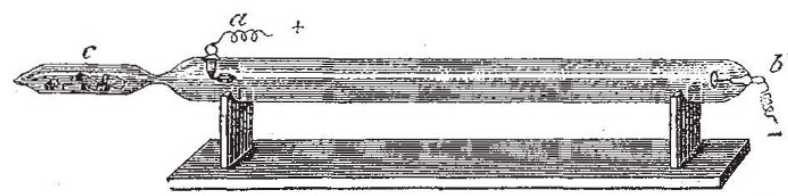

FIG. 5.

dependence of the phosphorescence or the glass on the degree of exhaustion. The two poles are at $a$ and $b$, and at the end $(c)$ is a sinall stipplementary tube, connected with the other by a narrow aperture, and containing solid caustic potash. The tube has been exhausted to a very high point, and the potash heated so as to drive off moisture and injure the vacuum. Exhaustion has then been recommenced, and the alternate heating and exhaustion repeated until the tube has been brought to the state in which it now appears before you. When the induction-spark is first turned on nothing is visible-the vacumm is so high that the tube is non-conducting. I now warm the potash slightiy, and liberate a trace of aqueous vapour. Instantly conduction commences, and the green phosphorescence flashes out along the lengtb of the tube. I continue the heat, so as to drive off more gas from the potash. The green gets fainter, and now a wave of cloudy luminosity sweeps over the tube, and stratifications appear, which rapidly get narrower, until the spark passes along the tube in the form of a narrow purple line. I take the lamp away, and allow the potash to cool; as it cools, the aqueous vapour, which the heat had driven off, is re-absorbed. The purple line broadens out, and breaks up into fine stratifications; these get wider, and travel towards the potash tube. Now a wave of green light appears on the glass at the other end, sweeping on and driving the last pale stratification into the potash;

$$
\begin{aligned}
& \begin{aligned}
x^{\circ} \circ \text { millionth of an atmosphere } & = \\
13 \times 5.789 \text { millionths of an atmosphere } & =\mathrm{r}^{\circ} 00076 \mathrm{millim} .
\end{aligned} \\
& 1,000,000 \\
& =760^{\circ} \text { o millims. }
\end{aligned}
$$

2 Nicarly roo years ago Mr. Wm. Morgan cominunicated to the Royal Society a paper entitled "Electrical Experiments made to ascertain the Non-conducting Power of a Perfect Vacuum, \&c." The following extracts from this paper, which was published in the Phil. Trans. for $I_{7} 8_{5}$ (vol. lxxv. Erom this paper, which was publishe
p. $27^{2}$ ), will be read with interest:-

p. $277^{2}$, will be read with interest:till every particle of air was expelled from the inside, was coated with tin-foil till every particle of air was expelled from the inside, was coated with tin-foil 5 inches down from its sealed end, and being inverted into mercury through a perforation in the brass cap which covered the mouth of the cistern; the
whole was cemented together, and the air was exhausted from the inside of the cistern through a valve in the brass cap, which, producing a perfect vacuum in the gage, formed an instrument peculiarly well adapted for experiments of this kind. Things being thus adjusted (a small wire having been previously fixed on the inside of the cistern to form a communication between the brass cap and the mercury, into which the gage was inverted the coated end was applied to the conductor of an electrical machine, and notwithstanding every effort, neither the sinallest ray of light, nor the slightest charge, could ever be procured in this exhausted gage.

"If the mercury in the gage be imperfectly boiled, the experiment will not succed; but the colour of the electric light, which, in air rarefied by an exhauster, is always violet or purple, appears in this case of a beautiful green, and, what is very curious, the degree of the air's rarefaction may be nearly determined by this means; for I have known instances, during the course of chese cxperiments, where a small particle of air, having found its way into the tuhe, the electric light became visible, and, as usual, of a green colour but the charge being often repeated, the gage has at length cracked at it sealed end, and in consequence the external air, by being admitted into th inside, has gradually produced a change in the electric light from green to blue, from blue to indigo, and so on to violet and purple, till the medium has at length become so dense as no longer to be a conductor of electricity. think there can be little doubt, from the above experiments, of the non-conducting power of a perfect vacuum.

"This seems to prove that there is a limit even in the rarefaction of air, which sets bounds to its conducting power; or, in other words, that the particles of air may be so far separated from each other as no longer to be able to transmit the electric fluid; and if they are brought within a cortain distance of each other their conducting power begins, and continually increases till their approach also arrives at its limit." and now the tube glows over its whole length with the green phosphorescence. I might keep it before you, and show the green growing fainter and the vacuum becoming non-conducting, but I should detain you too long, as time is required for the ab sorption of the last traces of vapour by the potash, and I must pass on to the next subject.

\section{Radiant Matter proceeds in straight Lines}

The radiant matter whose impact on the glass causes an evolution of light, absolutely refuses to turn a corner. Here is a V-shaped tube (Fig. 6), a pole being at each extremity. The pole at the right side $(a)$ being negative, you see that the whole of the right arm is flooded with green light, but at the bottom it stops sharply and will not turn the corner to get into the left side. When I reverse the current and make the left pole negative, the green changes to the left side, always following the negative pole and leaving the positive side with scarcely any luminosity.

In the ordinary phenomena exhibited by vacuum tubes-phe. nomena with which we are all familiar-it is customary, in order to bring out the striking contrasts of colour, to bend the tubes into very elaborate designs. The luminosity caused by the phos. phorescence of the residual gas follows all the convolutions into which skilful glass-blowers can manage to twist the glass. The

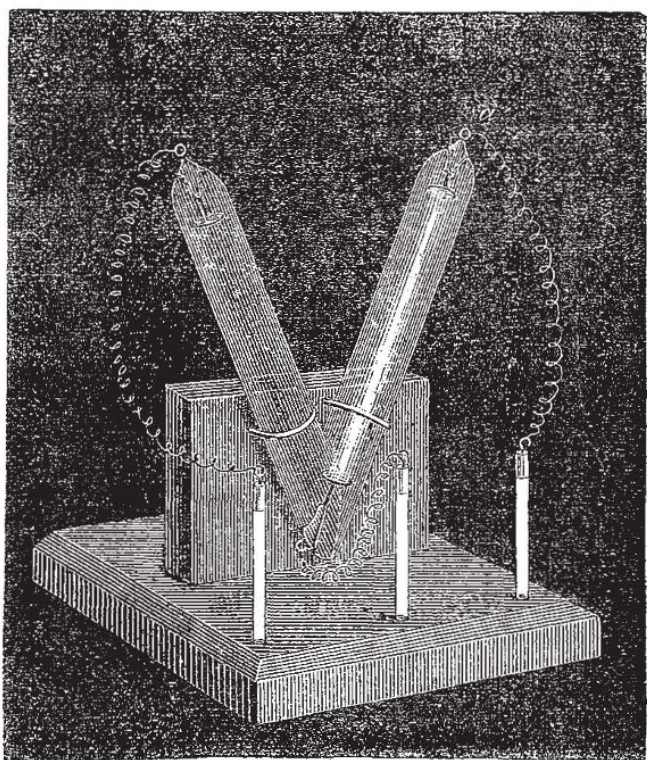

FIG. 6

negative pole being at one end and the positive pole at the other, the luminous phenomena seem to depend more on the positive than on the negative at the ordinary exhaustion hitherto used to get the best phenomena of vacuum tubes. But at a very high exhaustion the phenomena noticed in ordinary vacuum tubes when the induction spark passes through them-an appearance of cloudy luminosity and of stratifications-disappear entirely. No cloud or fog whatever is seen in the body of the tube, and with such a vacuum as I am working with in these experiments, the only light observed is that from the phosphorescent surface of the glass. I have here two bulbs (Fig. 7), alike in shape and position of poles, the only difference being that one is at an exhaustion equal to a few millimetres of mercury-such a moderate exhaustion as will give the ordinary luminous phenomena-whilst the other is exhausted to about the millionth of an atmosphere. I will first connect the moderately exhausted bulb (A) with the induction-coil, and retaining the pole at one side (a) always negative, I will put the positive wire successively to the other poles with which the bulb is furnished. You see that as I change the position of the positive pole, the line of violet light joining the two poles changes, the electric current always choosing the shortest path between the two poles, and moving about the bulb as I alter the position of the wires.

This, then, is the kind of phenomenon we get in ordinary exhaustions. I will now try the same experiment with a bulb (B) 
that is very highly exhausted, and as before, will make the side pole $\left(a^{\prime}\right)$ the negative, the top pole $(b)$ being positive. Notice how widely different is the appearance from that shown by the last bulb. The negative pole is in the form of a shallow cup. The molecular rays from the cup cross in the centre of the bulb, and thence diverging fall on the opposite side and produce a circular patch of green phosphorescent light. As I turn the bulb round you will all be able to see the green patch on the glass. Now observe, I remove the positive wire from the top, and connect it with the side pole (c). The green patch from the divergent negative focus is there still. I now make the lowest pole $(d)$ positive, and the green patch remains where it was at first, unchanged in position or intensity.

We have here another property of radiant matter. In the low vacuum the position of the positive pole is of every importance, whilst in a high vacuum the position of the positive pole scarcely matters at all; the phenomena seem to depend entirely on the negative pole. If the negative pole points in the direction of the

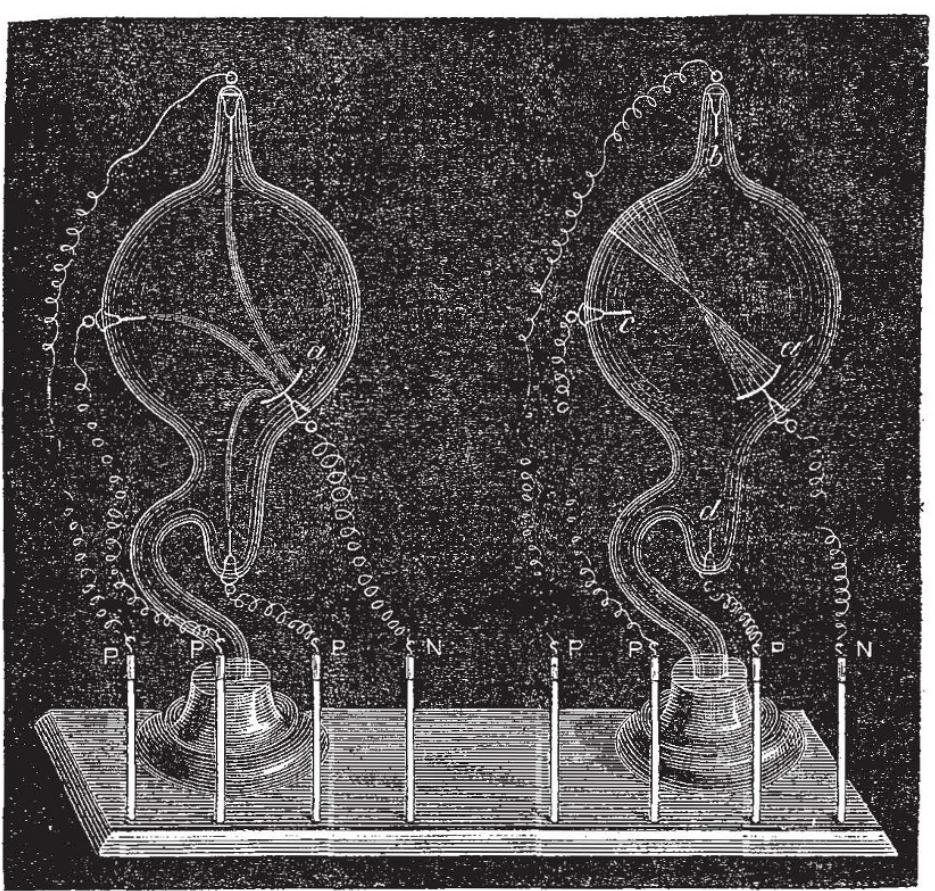

FIG. 7.

positive, all very well, but if the negative pole is entirely in the opposite direction it is of little consequence: the radiant matter darts all the same in a straight line from the negative.

If, instead of a flat disk, a hemi-cylinder is used for the negative pole, the matter still radiates normal to its surface. The tube before you (Fig. 8) illustrates this property. It contains, as a negative pole, a hemi-cylinder $(a)$ of polished aluminium. This is connected with a fine copper wire, $b$, ending at the platinum terminal, $c$. At the upper end of the tube is another terminal, $d$. The induction-coil is connected so that the hemicylinder is negative and the upper pole positive, and when exhausted to a sufficient extent the projection of the molecular rays to a focus is very beautifully shown. The rays of matter being driven from the hemi-cylinder in a direction normal to its surface, come to a focus and then diverge, tracing their path in brilliant green phosphorescence on the surface of the glass.

Instead of receiving the molecular rays on the glass, I will show you another tube in which the focus falls on a phosphorescent screen. See how brilliantly the lines of discharge shine out, and how intensely the focal point is illuminated, lighting up the table.

Radiant Matter when intercepted by Solid Matter casts a Shadow

Radiant matter comes from the pole in straight lines, and does not merely permeate all parts of the tube and fill it with light, as would be the case were the exhaustion less good. Where there is nothing in the way the rays strike the screen and produce ph-sphorescence, and where solid matter intervenes they are obstructed by it, and a shadow is thrown on the screen. In this pear-shaped bulb (Fig. 9) the negative pole $(a)$ is at the pointed end. In the middle is a cross $(b)$ cut out of sheet aluminium, so that the rays from the negative pole projected along the tube will be partly intercepted by the aluminium cross, and will project an image of it on the hemispherical end of the tube which is phosphorescent. I turn on the coil, and you will all see the black

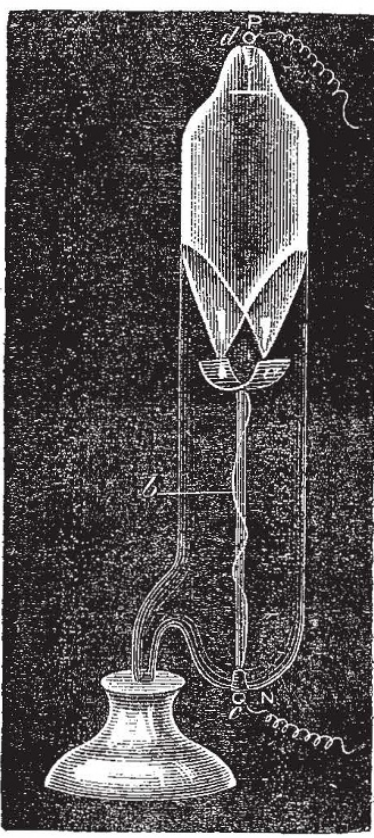

FIG. 8.

shadow of the cross on the luminous end of the bulb $(c, d)$. Now, the radiant matter from the negative pole has been passing by the side of the aluminium cross to produce the shadow; the glass has been hammered and bombarded till it is appreciably warm, and at the same time another effect has been produced on the glass-its sensibility has been deadened. The glass has got tired, if I may use the expression, by the enforced phosphorescence. A change has been produced by this molecular bom-

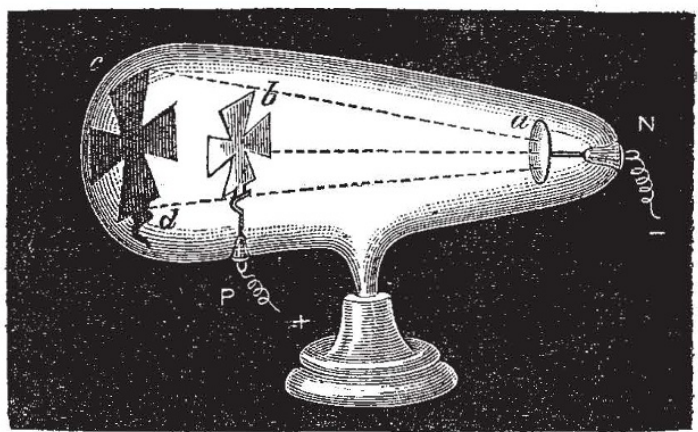

FIG. 9 .

bardment which will prevent the glass from responding easily to additional excitement; but the part that the shadow has fallen on is not tired-it has not been phosphorescing at all and is per. fectly fresh; therefore if I throw down this cross-I can easily do so by giving the apparatus a slight jerk, for it has been most ingeniously constructed with a hinge by Mr. Gimingham-and so allow the rays from the negative pole to fall uninterruptedly on to the end i $f$ the bulb, you will suddenly see the black cross 
$(c, d$, Fig. ro) change to a luminons one $(e, f)$, because the background is now only capable of faintly phosphorescing, whilst the part which had the black shadow on it retains its full phosphorescent power. The stencilled image of the luminous cross unfortunately soon dies out. After a period of rest the glass partly recovers its power of phosphorescing, but it is never so good as it waś at first.

Here, therefore, is another important property of radiant matter. It is projected with great velocity from the negative pole, and not only strikes the glass in such a way as to cause it to vibrate and become temporarily luminous while the discharge is going on, but the molecules hammer away with sufficient energy to produce a permanent impression upon the glass.

\section{(To be continued.)}

\section{NOTES}

IN accordance with the resolution come to at the recent International Congress of Meteorology, the International Committee have issued circulars for a special Conference at the Deutsche Seewarte at Hamburg, on October I, to consider the scherie of Count Wilczek and Lieut. Weyprecht for the establishment of circumpolar observing stations. The Conference will consider specially the following points:-I. The number of observatories and the most convenient places at which to establish them. The decision will depend on the number of co-operating states and the sums which they are willing to devote to this purpose. Count. Wilczek and Lieut. Weyprecht have proposed the following places:-In the Northern Hemisphere : north coasts of Spitzbergen and of Novaya Zemlya, the neighbourhoor of the North Cape, the mouth of the Lena, New Siberia, Point Barrow; on the north-east of Behring Strait, west coast of Greenland, east coast of Greenland, about $75^{\circ} \mathrm{N}$. lat. In the Southern Hemisphere: the neighbourhood of Cape Horn, Kerguelen or Macdonald Islands, one of the groups south of the Auckland Islands. 2. There will be considered the exact epoch of the observations and their maximum duration. 3. Uniform instruction for observations, which will have to fix especially: (a) The minimum of elements to be observed at each station, both for meteorological phenomena and for those of terrestrial magnetism, as well as for other phenomena of terrestrial physics connected with them. (b) The minimum number of daily observa. tions for the different elements. (c) The first meridian which will serve as basis for simultaneous observations. (d) Methods of observation for the different elements and methods of reduction. (e) Instruments of observation and their arrangement, as far as they may influence the comparability of the results.

AT a recent meeting of the Committee of the Iron and Steel Institute in Liverpool it was arranged that this year's meeting should be held in Liverpool on September 24, 25, and 26. The use of St. George's Hall has been granted by the Corporation, and numerous places for inspection and excursion have been partly arranged for, including. Messrs. Blundell's collieries, near Wigan, and the Tubular Bridge at Menai Straits. In addition to papers on the manufacture and application of steel and iron, papers on subjects of work more immediately connected with Eiverpool have been promised.

M. Janssen, we are glad to see, has been appointed to represent the Paris Academy of Sciences, at the inauguration of the statue to Arago, at Perpignan.

THE prizes instituted by Prof. Schäfli (Lausanne) for scientific works on Switzerland will now be awarded not only to Swiss naturalists, as hitherto, but also to foreign, a resolution in this sense having been accepted at the last meeting of Swiss naturalists.

WE regret to hear of the death of Mr. Edward Edwards, late of Menai Bridge, Anglesey, at the age of seventy-five. For upwards of twenty years he had studied the habits and characters of marine animals in their native haunts, and his contrivance of the "dark chamber tank" was the first by which these animal, could be kept alive and healthy for an indefinite period in confinement, and the principle of which was afterwards carefully recognised in the construction of the Crystal Palace and other aquariums.

THE Times Geneva correspondent writes, under date August 22 :- "On the evening of August 5, six persons who were standing in the gallery of a chalet in the Jura, above St. Cergues, witnessed an atmospheric phenomenon equally rare and curious. The aspect of the sky was dark and stormy. The air was thick with clouds, out of which darted at intervals bright flashes of lightning. At length one of these clouds, seeming to break loose from the mountains between - Nyon and the Dôle, advanced in the direction of a storm which had, meanwhile, broken out over Morges. The sun was hidden and the country covered with thick darkness. At this moment the pine forest round St. Cergues was suddenly illuminated and shone with a light bearing a striking resemblance to the phosphorescence of the sea as seen in the tropics. The light clisappeared with every slap of thunder, but only to re-appear with increased intensity until the subsidence of the tempest. M. Raoul Pictet, the eminent chemist, who was one of the witnesses of the phenomenon, thus explains it in the last number of the Archives des Sciences Physiques et Naturelles:- Before the appearance of this fire of St. Elmo, which covered the whole of the forest, it had rained several minutes during the first part of the storm. The rain had converted the trees into conductors of electricity. Then, when the cloud, strongly charged with the electric fluid, passed over this multitude of points, the discharges were sufficiently vivid to give rise to the luminous appearance. The effect was produced by the action of the electricity of the atmosphere on the electricity of the earth, an effect which, on the occasion in question, was considerably increased by the height of the locality, the proximity of a storm. clond, and the action of the rain, which turned all the trees of the forest into conductors.' "'

A young female gorilla is now being exhibited at the Crystal Palace.

AT the last meeting of the Swiss Naturalists, Prof. Kollmann (Basel) presented a report of the Anthropological and Statistical Commission, appointed by the Swiss Natural History Society for the investigation of the distribution of the light-coloured and dark-coloured population in Switzerland. Thanks to the collaboration of many schoolmasters, no less than 250,000 children in twenty-one cantons were described as to the colour of the eyes, hair, and skin, and a very rich and reliable material was collected. It is proved that in Switzerland, as well as in all middle Europe, the light-coloured population decreases from north to south, while the dark-coloured increases, and that it reaches its greatest quantity in the Graubünden, sending a rather dense branch to the south-west. It may be concluded that a dark-coloured population immigrated in Switzerland from the south, having also a side-branch which followed the direction from the Rhone to the Rhine.

WE are glad to learn that the great undertaking of printing and publishing a catalogue of the Advocates' Library, Edinburgh, which has been in progress for many years, is now approaching completion. The Library of the Faculty of Advocates ranks next to the British Museum and the Bodleian among the libraries of the United Kingdom. It contains about 262,000 printed volumes, besides manuscripts of great interest and importance. It has had (under the Copyright Act) since the reign of Queen Anne the right of receiving a copy of every book published in the United Kingdom. Last year there were added to the Library 4,007 volumes of books, besides periodicals, pamphlets, and 